\title{
Separation Theorems for Simplicity 26-Surfaces ${ }^{\star}$
}

\author{
J.C. Ciria, E. Domínguez, and A.R. Francés \\ Dpt. de Informática e Ingeniería de Sistemas. Facultad de Ciencias. \\ Universidad de Zaragoza. E-50009 - Zaragoza. Spain. \\ \{jcciria, afrances\}@posta. unizar.es
}

\begin{abstract}
The main goal of this paper is to prove a Digital JordanBrouwer Theorem and an Index Theorem for simplicity 26-surfaces. For this, we follow the approach to Digital Topology introduced in [2, and find a digital space such that the continuous analogue of each simplicity 26-surface is a combinatorial 2-manifold. Thus, the separation theorems quoted above turn out to be an immediate consequence of the general results obtained in [2] and 3 for arbitrary digital $n$-manifolds.
\end{abstract}

Keywords: Digital surface, simplicity 26 -surface, digital separation theorems.

\section{Introduction}

Several different notions of digital surface have been introduced on the grid $Z^{3}$, within the graph theoretical approach to Digital Topology, since Morgenthaler and Rosenfeld [9] defined the $(26,6)$ - and $(6,26)$-surfaces in 1981. Without going into all the existing notions, let us recall the $(\alpha, \beta)$-surfaces, for $\alpha, \beta \in\{6,18,26\}$ and $(\alpha, \beta) \neq(6,6)$, defined by Kong and Roscoe [7, which extend the original notion to other couples of adjacencies in $Z^{3}$, and the strong $n$-surfaces, $n \in$ $\{18,26\}$, of Bertrand and Malgouyres [6], where the set of strong 26-surfaces strictly contains all the (26,6)-surfaces (see [4]). More recently, Couprie and Bertrand [5] have introduced the notion of simplicity $n$-surface, for $n \in\{6,26\}$; and they have also shown that any strong 26 -surface is a simplicity 26 -surface.

Usually, some kind of separation theorem is obtained in order to show that these discrete objects are suitable digital counterparts to the notion of topological surface in the Euclidean space $\mathbb{R}^{3}$. For instance, in [7] and [6] it is shown that a digital version of the Jordan-Brouwer Theorem holds for $(\alpha, \beta)$-surfaces and strong 26-surfaces, respectively. Moreover, Morgenthaler and Rosenfeld show in [9] an Index Theorem for $(26,6)$ - and $(6,26)$-surfaces. However, similar results for simplicity 26-surfaces cannot yet be found in the literature. Our main goal in this paper is to prove a digital Jordan-Brouwer Theorem and an Index Theorem for these surfaces. These results have been found within the framework for Digital Topology introduced in [1] and [2].

* This work has been partially supported by the project DGES TIC2000-1368-C03-01.

A. Braquelaire, J.-O. Lachaud, and A. Vialard (Eds.): DGCI 2002, LNCS 2301, pp. 45 56 2002.

(C) Springer-Verlag Berlin Heidelberg 2002 
A digital space, as defined in $[2$, is a pair $(K, f)$ consisting of a polyhedral complex $K$, which represents the spatial layout of pixels, and a lighting function $f$ providing a "continuous interpretation" for each digital image in $K$. In this approach, a quite natural notion of digital $n$-dimensional manifold arises for which general digital versions of the Jordan-Brouwer and Index Theorems are stated and proved in arbitrary dimension (see 23]). Moreover, $(\alpha, \beta)$-surfaces and strong 26-surfaces turn out to be digital 2-manifolds (digital surfaces) for suitable digital spaces $\left(R^{3}, f_{\alpha \beta}\right)$ and $\left(R^{3}, f^{B M}\right)$, respectively, defined on the standard cubical decomposition of the Euclidean space $R^{3}$ (see [12]). This means, in particular, that the proof of such separation theorems can be directly applied to these kinds of surfaces. However, there does not exist a lighting function $f$ on the device model $R^{3}$ such that every simplicity 26 -surface is a digital surface in the digital space $\left(R^{3}, f\right)$. For this reason, in this paper, we consider a weaker version for one of the axioms defining lighting functions, in order to deal with a larger class of digital spaces for which the proofs of the general separation theorems quoted above still work. From these results, we derive in Section 3 a Jordan-Brouwer Theorem and an Index Theorem for simplicity 26-surfaces as follows. Firstly, we translate the notion of simplicity 26 -surface into terms of the device model $R^{3}$ (see Section 2). Then we find, also in Section[3, a lighting function $f^{\text {ss }}$ on $R^{3}$ satisfying the new weaker axiom, and we show that any simplicity 26 -surface is a digital surface in the digital space $\left(R^{3}, f^{\mathrm{ss}}\right)$. The long proof of this result is outlined in Section 4 while Section 1 introduces the basic notions of the approach to Digital Topology in 12 that are needed in this paper.

It is worth pointing out that simplicity 26 -surfaces are not the only digital surfaces in the space $\left(R^{3}, f^{\text {ss }}\right)$. At the present time, we do not know about the existence of a digital space whose digital surfaces coincide with the class of simplicity 26-surfaces. This will be the subject of a future work. On the other hand, the class of simplicity 6 -surfaces is the class of digital surfaces in the digital space $\left(R^{3}, f_{6,26}\right)$ given in [1]. This is a consequence of the fact that the former class coincides with Morgenthaler and Rosenfeld's $(6,26)$-surfaces $([5])$, and these are exactly the digital surfaces in $\left(R^{3}, f_{6,26}\right)$ (see [1]).

\section{Our Approach to Digital Topology}

The main purpose of Digital Topology is to study the topological properties of digital images. Digital images are discrete objects in nature, but they usually represent continuous objects or, at least, they are perceived as continuous objects. In our approach to Digital Topology [1, 2] we propose a multilevel architecture to represent, using different levels, each digital object together with the continuous perception that an observer may have of it, that we call its continuous analogue. In addition, several other intermediate levels allow us to relate the properties of digital objects with the corresponding properties of their continuous analogues. However, for simplicity, we introduce in this section only the levels of this architecture that are explicitly used in this paper. 
The first level, called device model, is a homogeneously $n$-dimensional locally finite polyhedral complex $K$, which is used to represent the spatial layout of space elements (xels for short). Each $n$-cell in a device model $K$ represents a xel, and so the digital object displayed in an image is a subset of the set $\operatorname{cell}_{n}(K)$ of $n$-cells in $K$; while the other lower dimensional cells in $K$ are used to describe how the xels could be linked to each other. In addition, the selection of a continuous analogue for each digital object in a device model $K$ is determined by a lighting function $f$ defined on $K$. So, a digital space is a pair $(K, f)$; that is, a device model $K$ together with a "continuous interpretation" for each digital object in $K$. Next we describe these two notions in detail.

By a homegeneously $n$-dimensional locally finite polyhedral complex we mean a set $K$ of polytopes, in some Euclidean space $\mathbb{R}^{d}$, provided with the natural ordering given by the relationship "to be face of", that in addition satisfies the four following properties:

1. If $\sigma \in K$ and $\tau$ is a face of $\sigma$ then $\tau \in K$.

2. If $\sigma, \tau \in K$ then $\sigma \cap \tau \in K$ is a face of both $\sigma$ and $\tau$.

3. For each point $x$ in the underlying polyhedron $|K|=\cup\{\sigma ; \sigma \in K\}$ of $K$, there exists a neighbourhood of $x$ which intersects only a finite number of polytopes in $K$; in particular, each polytope of $K$ is a face of a finite number of other polytopes in $K$.

4. Each polytope $\sigma \in K$ is a face of some $n$-dimensional polytope in $K$.

These complexes are particular cases of cellular complexes, as they are usually defined in polyhedral topology. So, in this paper, polytopes in $K$ will be simply referred to as cells, and $K$ itself will be called a polyhedral complex. The next paragraph recalls some elementary notions from polyhedral topology used in this paper. We refer to [10] for further notions on this subject.

Given a polyhedral complex $K$ and two cells $\gamma, \sigma \in K$, we shall write $\gamma \leq \sigma$ if $\gamma$ is a face of $\sigma$, and $\gamma<\sigma$ if in addition $\gamma \neq \sigma$. A centroid-map on $K$ is a map $c: K \rightarrow|K|$ such that $c(\sigma)$ belongs to the interior of $\sigma$; that is, $c(\sigma) \in \stackrel{\circ}{\sigma}=\sigma-\partial \sigma$, where $\partial \sigma=\cup\{\gamma ; \gamma<\sigma\}$ stands for the boundary of $\sigma$.

Remark 1. The device model $R^{n}$, called the standard cubical decomposition of the Euclidean $n$-space $\mathbb{R}^{n}$, is the complex determined by the collection of unit $n$-cubes in $\mathbb{R}^{n}$ whose edges are parallel to the coordinate axes and whose centers are in the set $\mathbb{Z}^{n}$. The centroid-map we will consider in $R^{n}$ associates each cube $\sigma$ with its barycentre $c(\sigma)$, which is a point in the set $\mathcal{Z}^{n}$. Here, $\mathcal{Z}=\frac{1}{2} \mathbb{Z}$ stands for the set of points $\{z \in \mathbb{R} ; z=y / 2, y \in \mathbb{Z}\}$. In particular, if $\operatorname{dim} \sigma=n$ then $c(\sigma) \in \mathbb{Z}^{n}$, where $\operatorname{dim} \sigma$ denotes the dimension of $\sigma$; and, thus, every digital object $O$ in $R^{n}$ can be identified with a subset of points in $\mathbb{Z}^{n}$.

Before proceeding with the definition of lighting function, we need to introduce the following notions.

The first two notions formalize two types of "digital neighbourhoods" of a cell $\alpha \in K$ in a given digital object $O \subseteq \operatorname{cell}_{n}(K)$. Indeed, the star of $\alpha$ in $O$ is the set $\operatorname{st}_{n}(\alpha ; O)=\{\sigma \in O ; \alpha \leq \sigma\}$ of $n$-cells (xels) in $O$ having $\alpha$ as a face. 
Similarly, the extended star of $\alpha$ in $O$ is the set $\operatorname{st}_{n}^{*}(\alpha ; O)=\{\sigma \in O ; \alpha \cap \sigma \neq \emptyset\}$ of $n$-cells (xels) in $O$ intersecting $\alpha$. Finally, the support of a digital object $O$ is the set $\operatorname{supp}(O)$ of cells of $K$ (not necessarily xels) that are the intersection of $n$-cells (xels) in $O$. Namely, $\alpha \in \operatorname{supp}(O)$ if and only if $\alpha=\cap\left\{\sigma ; \sigma \in \operatorname{st}_{n}(\alpha ; O)\right\}$. Notice that $O \subseteq \operatorname{supp}(O)$ for any digital object $O$, and the elements in $\operatorname{supp}(O)-O$ are the lower dimensional cells of $K$ that directly connect at least two xels in $O$.

For ease of writing, we use the following notation: $\operatorname{supp}(K)=\operatorname{supp}\left(\operatorname{cell}_{n}(K)\right)$, $\operatorname{st}_{n}(\alpha ; K)=\operatorname{st}_{n}\left(\alpha ; \operatorname{cell}_{n}(K)\right)$ and $\operatorname{st}_{n}^{*}(\alpha ; K)=\operatorname{st}_{n}^{*}\left(\alpha ; \operatorname{cell}_{n}(K)\right)$. Finally, we shall write $\mathcal{P}(A)$ for the family of all subsets of a given set $A$.

Definition 1. Given a device model $K$, a lighting function on $K$ is a map $f: \mathcal{P}\left(\operatorname{cell}_{n}(K)\right) \times K \rightarrow\{0,1\}$ satisfying the following five axioms for all $O \in$ $\mathcal{P}\left(\operatorname{cell}_{n}(K)\right)$ and $\alpha \in K$ :

1. object axiom: if $\operatorname{dim} \alpha=\operatorname{dim} K$, then $f(O, \alpha)=1$ if and only if $\alpha \in O$;

2. support axiom: if $\alpha \notin \operatorname{supp}(O)$ then $f(O, \alpha)=0$;

3. monotone axiom: $f(O, \alpha) \leq f\left(\operatorname{cell}_{n}(K), \alpha\right)$;

4. local axiom: $f(O, \alpha)=f\left(\operatorname{st}_{n}^{*}(\alpha ; O), \alpha\right)$; and,

5. complement connectivity axiom: if $O^{\prime} \subseteq O \subseteq \operatorname{cell}_{n}(K)$ and $\alpha \in K$ are such that $\operatorname{st}_{n}(\alpha ; O)=\operatorname{st}_{n}\left(\alpha ; O^{\prime}\right), f\left(O^{\prime}, \alpha\right)=0$ and $f(O, \alpha)=1$, then: (a) the set of cells $\alpha\left(O^{\prime} ; O\right)=\left\{\omega<\alpha ; f\left(O^{\prime}, \omega\right)=0, f(O, \omega)=1\right\}$ is not empty; and, (b) the set $\cup\left\{\stackrel{\dot{\omega}}{\omega} \omega \in \alpha\left(O^{\prime} ; O\right)\right\}$ is connected in $\partial \alpha$.

If $f(O, \alpha)=1$ we say that $f$ lights the cell $\alpha$ for the object $O$.

As quoted above, the lighting function $f$ provides a continuous interpretation for each digital object in a digital space $(K, f)$, which is represented by an Euclidean polyhedron in the fifth level of our architecture. To introduce these polyhedra we use an arbitrary but fixed centroid-map $c: K \rightarrow|K|$ on the device model $K$.

Definition 2. Let $O \subseteq \operatorname{cell}_{n}(K)$ be a digital object in the digital space $(K, f)$. The continuous analogue of $O$ is the underlying polyhedron $\left|\mathcal{A}_{O}\right|$ of the simplicial complex $\mathcal{A}_{O}$, whose $k$-simplexes are $\left\langle c\left(\alpha_{0}\right), c\left(\alpha_{1}\right), \ldots, c\left(\alpha_{k}\right)\right\rangle$ where $\alpha_{0}<\alpha_{1}<$ $\cdots<\alpha_{k}$ are cells in $K$ such that $f\left(O, \alpha_{i}\right)=1$, for $0 \leq i \leq k$. The complex $\mathcal{A}_{O}$ is called the simplicial analogue of $O$.

Since continuous analogues represent the continuous interpretation that an observer makes of digital objects, our architecture allows us to introduce digital notions in terms of the corresponding continuous ones. For example, we will say that an object $O$ is connected if its continuous analogue $\left|\mathcal{A}_{O}\right|$ is a connected polyhedron. Similarly, if we consider the set of xels $\operatorname{cell}_{n}(K)-O$ as the complement of the digital object $O$, we say that $\operatorname{cell}_{n}(K)-O$ is connected if the complement of the object's continuous analogue $\left|\mathcal{A}_{\text {cell }_{n}(K)}\right|-\left|\mathcal{A}_{O}\right|$ is a connected topological space. And, an object $O$ is also naturally called a $m$-dimensional digital manifold if $\left|\mathcal{A}_{O}\right|$ is a combinatorial $m$-manifold without boundary; that is, we call $O$ a digital surface (2-manifold) if it looks like a surface. However, doing 
this, the problem of characterizing these notions in digital terms arises (see [2] for connectivity, and 1], [2] for digital surfaces).

But, even more interesting than the definition of new digital notions is the possibility of translating results from polyhedral topology, through the levels of this architecture, in order to obtain general results in Digital Topology. Two examples of this powerful technique are the Digital Jordan-Brouwer and Index Theorems for arbitrary $m$-manifolds proved in 2] and [3], respectively, which generalize the well-known result of Morgenthaler and Rosenfeld ([9]) to all types of $(\alpha, \beta)$-surfaces $([7])$ and to the strong 26 -surfaces $([6])$. At this point, it is worth pointing out that, sometimes, the continuous result can be translated into digital terms directly, as was the case of the Digital Jordan-Brouwer Theorem; but, in general, some previous work must be done at the continuous level to adapt the continuous result before the translation can be carried out.

In this paper we consider, however, a class of digital spaces larger than the class for which these separation theorems were proved in [2] and [3]. The lighting functions defining this restricted class of spaces satisfy, in addition to the five axioms in Definition 1, the following condition

$$
\begin{aligned}
& \text { Subspace condition: Let } O^{\prime} \subseteq O \subseteq \operatorname{cell}_{n}(K) \text { be two digital objects } \\
& \text { and } \alpha \in K \text { a cell such that } \operatorname{st}_{n}(\alpha ; O)=\operatorname{st}_{n}\left(\alpha ; O^{\prime}\right), f\left(O^{\prime}, \alpha\right)=0 \\
& \text { and } f(O, \alpha)=1 \text {. Then, if } O \subseteq O \subseteq \operatorname{cell}_{n}(K), f(\bar{O}, \beta)=1 \text { for every } \\
& \beta \in \alpha\left(O^{\prime} ; O\right) \text {. }
\end{aligned}
$$

This is a sufficient condition to ensure that each digital object $O$ in a digital space $(K, f)$ induces a digital subspace $\left(K(O), f_{O}\right)$, where $K(O)=\{\alpha \in K ; \alpha \leq \sigma \in$ $O\}$ is the subcomplex of $K$ induced by the cells in $O$ and the lighting function $f_{O}$ is defined by $f_{O}\left(O^{\prime}, \alpha\right)=f(O, \alpha) f\left(O^{\prime}, \alpha\right)$ for every $O^{\prime} \subseteq O$ and $\alpha \in K(O)$. It is not difficult to observe that the proofs given in [2] and 3] do not make use of this additional condition anywhere; and, thus, the Jordan-Brouwer and Index Theorems hold for the extended class of digital spaces considered in this paper.

\section{About Simplicity 26-Surfaces}

Couprie and Bertrand [5] define a simplicity 26-surface as a "thin" subset of $Z^{3}$ such that a certain neighbourhood of each one of its points constitutes a simple closed curve under the structure of a particular graph, which is defined using the notions of homotopy and simple point. In the same paper [5], two local characterizations of simplicity 26-surfaces are provided. One of them states that any simplicity 26-surface can be obtained by concatenating a certain family of patterns according to a number of rules; in particular, the patterns around each point must define a cycle. In this paper we will use this characterization as the definition of simplicity 26-surfaces.

Before introducing this definition of simplicity 26-surfaces in terms of digital objects in the device model $R^{3}$, we firstly recall, in this language, some basic notions of the graph-theoretical approach to Digital Topology. For this, we use the identification between the sets $Z^{3}$ and $\operatorname{cell}_{3}\left(R^{3}\right)$ given in Remark 1 . 
Given a xel $\sigma \in \operatorname{cell}_{3}\left(R^{3}\right)$ we consider the following three neighbourhoods $([8]): N_{26}(\sigma)=\left\{\tau \in \operatorname{cell}_{3}\left(R^{3}\right) ; \sigma \cap \tau \neq \emptyset\right\}, N_{18}(\sigma)=\left\{\tau \in N_{26}(\sigma) ; \operatorname{dim} \sigma \cap\right.$ $\tau \geq 1\} \cup\{\sigma\}$, and $N_{6}(\sigma)=\left\{\tau \in N_{26}(\sigma) ; \operatorname{dim} \sigma \cap \tau=2\right\} \cup\{\sigma\}$. Notice that $N_{26}(\sigma)=\operatorname{st}_{3}^{*}\left(\sigma ; R^{3}\right)$. Two xels $\sigma, \tau \in \operatorname{cell}_{3}\left(R^{3}\right)$ are said to be $n$-adjacent if $\tau \in N_{n}^{*}(\sigma)=N_{n}(\sigma) \backslash\{\sigma\}$, where $n \in\{6,18,26\}$. Given a digital object $O \subseteq \operatorname{cell}_{3}\left(R^{3}\right)$ and two xels $\sigma, \tau \in O$, a $n$-path in $O$ from $\sigma$ to $\tau$ is a sequence $\left(\sigma_{i}\right)_{i=0}^{k} \subseteq O$ such that $\sigma_{0}=\sigma, \sigma_{k}=\tau$, and $\sigma_{i-1}$ is $n$-adjacent to $\sigma_{i}$, for $1 \leq i \leq k$. Notice that $n$-paths define an equivalence relation in the object $O$, whose classes are called the $n$-components of $O$. Finally, a digital object $O \subseteq \operatorname{cell}_{3}\left(R^{3}\right)$ is said to be $n$-connected if it consists of only one $n$-component.

Next, we introduce the family of patterns defining simplicity 26 -surfaces. For this we need the following

Definition 3. Let $O \subseteq \operatorname{cell}_{3}\left(R^{3}\right)$ be a digital object. A vertex $\alpha \in R^{3}$ is said to be maximal with respect to $O$ if $\operatorname{st}_{3}(\alpha ; O) \neq \emptyset$ and, in addition, $\operatorname{st}_{3}(\alpha ; O)=\operatorname{st}_{3}(\beta ; O)$ for any other vertex $\beta \in R^{3}$ such that $\operatorname{st}_{3}(\alpha ; O) \subseteq \operatorname{st}_{3}(\beta ; O)$. If $\alpha$ is maximal with respect to $O$, we say that $\operatorname{st}_{3}(\alpha ; O)$ is a maximal trace (or simply a trace) of $O$.

Given a digital object $O \subseteq \operatorname{cell}_{3}\left(R^{3}\right)$, the set of traces of $O$ will be denoted by $\operatorname{Tr}(O)$, while $\operatorname{Tr}(\sigma, O)$ will stand for the traces of $O$ containing a given xel $\sigma \in O$.

Couprie and Bertrand define in [5] a graph on the set of traces of a given digital object as follows.

Definition 4 (Def. 16 in [5]). Let $O \subseteq \operatorname{cell}_{3}\left(R^{3}\right)$ be a digital object. The vertices of the graph $\operatorname{GTr}(O)$ are the traces of $O$, and two distinct traces $t_{1}, t_{2} \in$ $\operatorname{Tr}(O)$ are adjacent in $\operatorname{GTr}(O)$ if the intersection $t_{1} \cap t_{2}$ consists of exactly two elements.

Remark 2 (Lemma 15 in [5]). The traces of an arbitrary digital object $O$ may correspond (up to rotations and symmetries) to 21 different patterns. However, only the eight patterns from the set $A_{26}=\left\{a_{1}, \ldots, a_{8}\right\}$ depicted in Fig. 1 may appear in a simplicity 26 -surface.

Definition 5 (Th. 17 in [5]). A 26-connected digital object $S \subseteq \operatorname{cell}_{3}\left(R^{3}\right)$ is said to be a simplicity 26-surface if the following conditions are satisfied for any xel $\sigma \in S$ :

1. $T_{26}(\sigma, S)=1$ and $T_{6}\left(\sigma, \operatorname{cell}_{3}\left(R^{3}\right)-S\right)=2$.

2. Every trace of $S$ corresponds (up to rotations and symmetries) to an element of the set $A_{26}$.

3. $\sigma$ is the only xel in $S$ belonging to all of the traces of $\operatorname{Tr}(\sigma, S)$; that is, $\sigma=\cap\{t ; t \in \operatorname{Tr}(\sigma, S)\}$.

4. For any two distinct traces $t_{1}, t_{2} \in \operatorname{Tr}(\sigma, S), t_{1} \cap t_{2}$ has at most two elements.

5. The set of traces $\operatorname{Tr}(\sigma, S)$ constitutes a simple closed curve in the graph $\operatorname{GTr}(O)$; that is, for each trace $t \in \operatorname{Tr}(\sigma, O)$ there exist exactly two other traces $t_{1}, t_{2} \in \operatorname{Tr}(\sigma, O)$ which are adjacent to $t$ in the graph $\operatorname{GTr}(\sigma, O)$. 


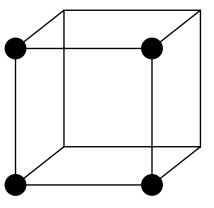

$a_{1}$

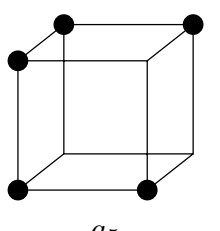

$a_{5}$

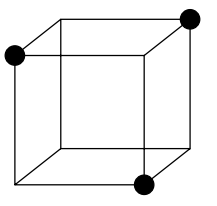

$a_{2}$

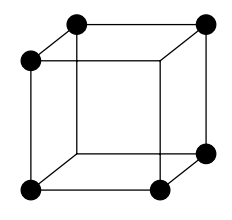

$a_{6}$

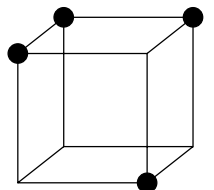

$a_{3}$

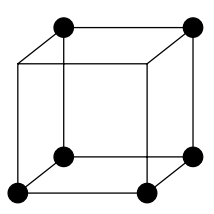

$a_{7}$

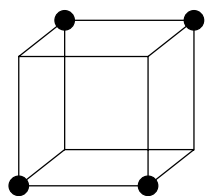

$a_{4}$

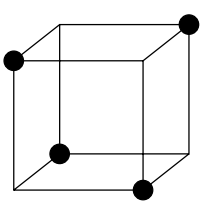

$a_{8}$

Fig. 1. The eight patterns defining simplicity 26 -surfaces.

\section{Main Results}

As was quoted above, our main goal in this paper is to state and prove a JordanBrouwer Theorem and an Index Theorem for simplicity 26-surfaces. For this purpose, we firstly find a digital space in which the continuous analogue of each simplicity 26-surface is a combinatorial surface. This space is defined on the device model $R^{3}$, the standard cubical decomposition of the Euclidean 3-space $\mathbb{R}^{3}$, by the lighting function $f^{\mathrm{ss}}$, which is given by $f^{\mathrm{ss}}(O, \alpha)=1$ if and only if: (a) $\operatorname{dim} \alpha=3$ and $\alpha \in O$; (b) $\operatorname{dim} \alpha=2$ and $\alpha \in \operatorname{supp}(O)$; (c) $\operatorname{dim} \alpha=0$, $\alpha \in \operatorname{supp}(O)$ but $\operatorname{st}_{3}(\alpha ; O)$ does not correspond to the pattern $a_{7}$ in Fig. If; and (d) $\operatorname{dim} \alpha=1$ and one of the two following conditions holds:

(d1) $\operatorname{st}_{3}\left(\alpha ; R^{3}\right) \subseteq O$

(d2) $\operatorname{st}_{3}(\alpha ; O)=\left\{\sigma_{1}, \sigma_{2}\right\}$, with $\alpha=\sigma_{1} \cap \sigma_{2}$, and the two vertices $\beta_{1}$ and $\beta_{2}$ of $\alpha$ are both maximal with respect to $O$.

Next we give several immediate properties of the digital space $\left(R^{3}, f^{\text {ss }}\right)$ needed in the sequel.

Remark 3. 1) For any cell $\alpha \in R^{3}, f^{\mathrm{ss}}\left(\operatorname{cell}_{3}\left(R^{3}\right), \alpha\right)=1$. So that, the continuous analogue of the digital object $\operatorname{cell}_{3}\left(R^{3}\right)$ consisting of all the xels in $\left(R^{3}, f^{\mathrm{ss}}\right)$ is $\left|\mathcal{A}_{\text {cell }_{3}\left(R^{3}\right)}\right|=\mathbb{R}^{3}$ the Euclidean 3 -space.

2) The digital space $\left(R^{3}, f^{\mathrm{ss}}\right)$ is solid; that is, $f^{\mathrm{ss}}(O, \alpha)=1$ for any cell $\alpha \in R^{3}$ and any digital object $O \subseteq \operatorname{cell}_{3}\left(R^{3}\right)$ such that $\operatorname{st}_{3}\left(\alpha ; R^{3}\right) \subseteq O$.

3) The digital space $\left(R^{3}, f^{\mathrm{ss}}\right)$ is strongly local at dimensions 0,2 and 3 ; that is, if $\operatorname{dim} \alpha \in\{0,2,3\}$ then $f^{\mathrm{ss}}(O, \alpha)=f^{\mathrm{ss}}\left(\operatorname{st}_{3}(\alpha ; O), \alpha\right)$ for any object $O$.

Now we are ready to state our basic result, whose long and quite technical proof is outlined in Section 4.

Theorem 1. Every simplicity 26-surface is a digital surface in the digital space $\left(R^{3}, f^{\mathrm{ss}}\right)$; that is, the continuous analogue $\left|\mathcal{A}_{S}\right|$ of any simplicity 26-surface $S$ is a combinatorial surface. 


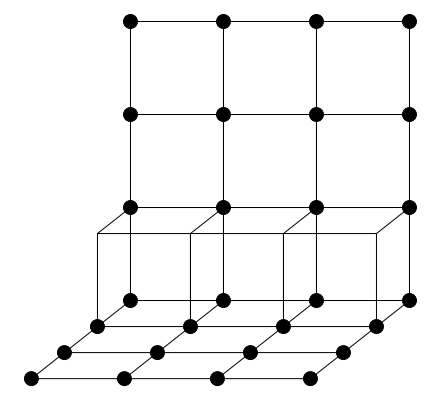

Fig. 2. A digital surface in $\left(R^{3}, f^{\text {ss }}\right)$ which is not a simplicity 26-surface.

Remark 4. Simplicity 26-surfaces are not the only digital surfaces in the digital space $\left(R^{3}, f^{\text {ss }}\right)$. For example, it is easy to check that the digital object $S$ depicted in Fig. 2 is a digital surface. However, it is not a simplicity 26-surface since any two traces in $S$ corresponding to the pattern $a_{7}$ meet in three xels.

Both the Jordan-Brouwer Theorem and the Index Theorem are related to the connectivity of the complement of digital objects; so that, we need to determine the connectivity associated to the digital space $\left(R^{3}, f^{\mathrm{ss}}\right)$. This is done in the next result, whose proof is straightforward from the definition of the lighting function $f^{\text {ss }}$ and the characterization of the connectivity of an object and its complement given in [2, Theorem 4.2] (see also [1]).

Proposition 1. Let $O$ be a digital object in $\left(R^{3}, f^{\mathrm{ss}}\right)$. Then

1. a susbset $C \subseteq O$ is a 26-component of $O$ if and only if $C=\{\sigma \in O ; c(\sigma) \in$ $X\}$ for some component $X$ of the continuous analogue $\left|\mathcal{A}_{O}\right|$ of $O$; and

2. a susbset $D \subseteq \operatorname{cell}_{3}\left(R^{3}\right)-O$ is a 6-component of $\operatorname{cell}_{3}\left(R^{3}\right)-O$ if and only if $D=\{\sigma \in O ; c(\sigma) \in Y\}$ for some component $Y$ of the complement $\mathbb{R}^{3}-$ $\left|\mathcal{A}_{O}\right|=\left|\mathcal{A}_{\mathrm{cell}_{3}\left(R^{3}\right)}\right|-\left|\mathcal{A}_{O}\right|$ of the continuous analogue of $O$.

As quoted in Section[1, the Digital Jordan-Brouwer and Index Theorems were originally proved in [2. Theorem 5.3] and [3, Theorem 3.16], respectively, for the class of digital spaces satisfying the subspace condition. However, these proofs do not make use of such condition. Thus, to reach our goal, it will suffice to check that the hypotheses of these general separation theorems are satisfied in the digital space $\left(R^{3}, f^{\text {ss }}\right)$, which is actually done in Remarks 3(1) and (2). In this way, the Jordan-Brouwer Theorem for simplicity 26-surfaces follows from the next result, which is an immediate consequence of Proposition 1 and Theorem 5.3 in 2 .

Theorem 2 (Jordan-Brouwer Theorem). Let $S$ be a 26-connected digital surface in $\left(R^{3}, f^{\mathrm{ss}}\right)$. Then, its complement cell ${ }_{3}\left(R^{3}\right)-S$ consists of two 6 components. Moreover, if $S$ is finite then one of the 6-components of $\operatorname{cell}_{3}\left(R^{3}\right)-S$ is also finite. 
To state the Index Theorem for simplicity 26-surfaces we need the following additional notions (see 3] for details).

Let $\sigma \in \operatorname{cell}_{3}\left(R^{3}\right)$ be a xel with centroid $c(\sigma)=\left(x_{1}, x_{2}, x_{3}\right) \in \mathbb{Z}^{3}$. We call the object $H_{\sigma}=\left\{\tau \in \operatorname{cell}_{3}\left(R^{3}\right) ; c(\tau)=\left(x_{1}, x_{2}, \lambda\right) \in \mathbb{Z}^{3}, \lambda \geq x_{3}\right\}$ a digital half-line starting at $\sigma$. Given a finite digital surface $S$ in $\left(R^{3}, f^{\mathrm{ss}}\right)$ such that $\sigma \notin S$, the 26-components of $H_{\sigma} \cap S$ turn out to be finite digital segments; and thus, we can consider the predecessor $\pi_{C}$ and the successor $\sigma_{C}$ of $C$ in $H_{\sigma}$. Then, we say that the half-line $H_{\sigma}$ meets $S$ transversally at $C$ if $\pi_{C}$ and $\sigma_{C}$ lie in the same 6-component of the object $\cup_{\sigma \in C} N_{26}(\sigma)-S$.

Now, Proposition 1 and Theorem 3.16 in [3] yield the following result.

Theorem 3 (Index Theorem). Let $S$ be a finite digital surface in $\left(R^{3}, f^{\mathrm{ss}}\right)$. $A$ xel $\sigma \notin S$ belongs to the finite 6-component of the complement of $S$ if and only if the half-line $H_{\sigma}$ meets $S$ transversally an odd number of times.

\section{An Outline of the Proof of Theorem 1}

Our goal in this Section is to prove that, for any simplicity 26-surface $S$, the continuous analogue $\left|\mathcal{A}_{S}\right|$ in the digital space $\left(R^{3}, f^{\text {ss }}\right)$ is a combinatorial surface. For this we will find a suitable cellular decomposition of the polyhedron $\left|\mathcal{A}_{S}\right|$.

By a (principal) cellular decomposition of a polyhedron $P$ we mean a triple $\mathcal{C}=(\mathcal{F}, \mathcal{E}, \mathcal{P})$ of families of faces (2-balls), edges (1-balls) and points (0-balls), respectively, satisfying the following properties:

1. for any two distinct faces $f_{1}, f_{2} \in \mathcal{F}$, their intersection $f_{1} \cap f_{2}$ is a ball in $\mathcal{E} \cup \mathcal{P}$ or it is the empty set;

2. for any two distinct edges $e_{1}, e_{2} \in \mathcal{E}$, the intersection $e_{1} \cap e_{2}$ is a point in $\mathcal{P}$ or it is empty;

3. the polyhedron $P$ is the union of all balls in $\mathcal{F} \cup \mathcal{E} \cup \mathcal{P}$; and,

4. each point $p \in \mathcal{P}$ is contained in some edge $e \in \mathcal{E}$; and, similarly, each edge $e \in \mathcal{E}$ is contained in some face $f \in \mathcal{F}$.

A polyhedron $P$ is said to be a combinatorial surface if it has a cellular decomposition $\mathcal{C}=(\mathcal{F}, \mathcal{E}, \mathcal{P})$ for which the two following additional conditions holds:

5. every edge $e \in \mathcal{E}$ is included in exactly two faces; and,

6. for every point $p \in \mathcal{P}$, the union $\cup\{f \in \mathcal{F} ; p \subseteq f\}$ is a 2-ball.

We claim that, for any simplicity 26-surface, there exists a cellular decomposition $\mathcal{C}_{S}=\left(\mathcal{F}_{S}, \mathcal{E}_{S}, \mathcal{P}_{S}\right)$ of its continuous analogue $\left|\mathcal{A}_{S}\right|$ satisfying the five properties above. The triple $\mathcal{C}_{S}$ is defined as follows:

- the faces in $\mathcal{F}_{S}$ are the polyhedra $f_{\alpha}=\left|\mathcal{A}_{S}\right| \cap\left|\mathcal{A}_{\text {st }_{3}\left(\alpha ; R^{3}\right)}\right|$, where $\alpha$ ranges over the set of vertices of $R^{3}$ which are maximal with respect to $S$;

- the edges in $\mathcal{E}_{S}$ are the intersections $e_{\alpha \beta}=f_{\alpha} \cap f_{\beta}$, where $f_{\alpha}, f_{\beta}$ are two distinct faces in $\mathcal{F}_{S}$ such that the traces $\operatorname{st}_{3}(\alpha ; S)$ and $\operatorname{st}_{3}(\beta ; S)$ share exactly two xels of $S$; and, finally,

- the family $\mathcal{P}_{S}$ of points is the set of centroids $c(\sigma)$ where $\sigma \in S$. 

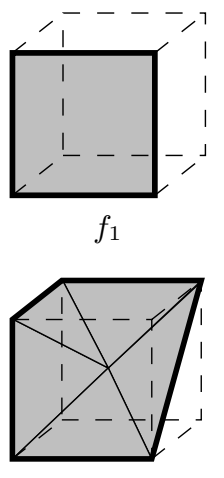

$f_{5}$

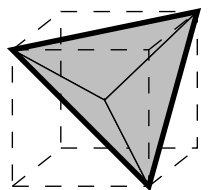

$f_{2}$

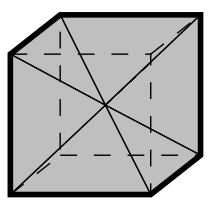

$f_{6}$

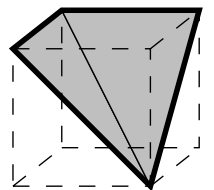

$f_{3}$

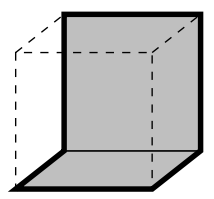

$f_{7}$

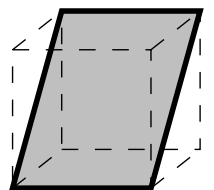

$f_{4}$

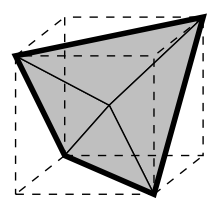

$f_{8}$

Fig. 3. The eight faces of the cellular decomposition of $\left|\mathcal{A}_{S}\right|$. The bold segments are the edges in $\mathcal{E}_{S}$ contained in these faces.

In the rest of this Section we give an outline of the long and rather technical proof of our claim. The first step is to determine the shape of the faces in the set $\mathcal{F}_{S}$, which is done in the following result.

Proposition 2. Let $S$ be a simplicity 26-surface in $\left(R^{3}, f^{\mathrm{ss}}\right)$ and $\alpha \in R^{3}$ a maximal vertex with respect to $S$. If the trace $\operatorname{st}_{3}(\alpha ; S)$ corresponds to the pattern $a_{i}(1 \leq i \leq 8)$ in Fig. [1, then the face $f_{\alpha}=\left|\mathcal{A}_{S}\right| \cap\left|\mathcal{A}_{\mathrm{st}_{3}\left(\alpha ; R^{3}\right)}\right|$ is (up to rotations and symmetries) the 2-ball $f_{i}$ depicted in Fig. 3 .

The proof of Proposition 2 makes use of the fact that the simplicial analogues $\mathcal{A}_{S}$ and $\mathcal{A}_{\mathrm{st}_{3}\left(\alpha ; R^{3}\right)}$ are both full subcomplexes of the first derived subdivision of the device model $R^{3}$, which is determined by the centroid-map $c: R^{3} \rightarrow\left|R^{3}\right|$ that associates each cell $\gamma \in R^{3}$ with its barycentre (see Remark 1). Moreover, the vertices of $\mathcal{A}_{\mathrm{st}_{3}\left(\alpha ; R^{3}\right)}$ are the centroids $c(\gamma)$ of all cells $\gamma \in R^{3}$ having $\alpha$ as a face. Thus, the 2 -ball $f_{\alpha}$ is determined by the set of cells $\left\{\gamma \in R^{3} ; \alpha \leq\right.$ $\gamma$ and $\left.f^{\mathrm{ss}}(S, \gamma)=1\right\}$. To characterize this set we distinguish two cases: $\operatorname{dim} \gamma \in$ $\{0,2,3\}$ and $\operatorname{dim} \gamma=1$.

The following result, which is almost immediate from Remark 3(3), states that, in the first case, the cell $\gamma$ is lighted for the simplicity 26-surface $S$ if and only if it is lighted for the trace determined by $\alpha$.

Proposition 3. Let $S$ be a simplicity 26-surfaces and $\alpha \in R^{3}$ a maximal vertex with respect to $S$. If $\alpha \leq \gamma$ and $\operatorname{dim} \gamma \in\{0,2,3\}$, then $f^{\mathrm{ss}}(S, \gamma)=$ $f^{\mathrm{ss}}\left(\operatorname{st}_{3}(\alpha ; S), \gamma\right)$.

If $\operatorname{dim} \gamma=1$, it suffices, by the definition of the lighting function $f^{\text {ss }}$, to analyze the case $\operatorname{st}_{3}(\gamma ; S)=\left\{\tau_{1}, \tau_{2}\right\}$ with $\gamma=\tau_{1} \cap \tau_{2}$. For this, we use the pattern corresponding to the trace $\operatorname{st}_{3}(\alpha ; S)$ to consider two subcases, which are summarized in the next result. 
Proposition 4. Let $S$ be a simplicity 26-surfaces and $\alpha \in R^{3}$ a maximal vertex with respect to $S$ such that the trace $\operatorname{st}_{3}(\alpha ; S)$ does not correspond to the pattern $a_{8}$ in Fig. 1. Then, $f^{\mathrm{ss}}(S, \gamma)=1$ for any 1-cell $\alpha \leq \gamma$ such that $\operatorname{st}_{3}(\gamma ; S)=$ $\left\{\tau_{1}, \tau_{2}\right\}$ with $\gamma=\sigma \cap \tau$. On the other hand, if the trace $\operatorname{st}_{3}(\alpha ; S)=\left\{\sigma, \sigma_{1}, \sigma_{2}, \sigma_{3}\right\}$ corresponds to the pattern $a_{8}$, then exactly one of the three 1-cells $\gamma_{i}^{\sigma}=\sigma \cap \sigma_{i}$, $i=1,2,3$, is not lighted for $S$.

Now, it is not difficult to show Proposition 2 from Propositions 3 and 4. Moreover, we derive from the definition of the family $\mathcal{E}_{S}$ that the edges $e_{\alpha \beta}$ are the bold segments in Fig. 3. Here, the only tricky point occurs when the trace $t=\operatorname{st}_{3}(\alpha ; S)$ corresponds to the pattern $a_{7}$. But, in this case is possible to show that $t$ is the only trace of $S$ containing the pair $\{\sigma, \tau\}$, where the points $c(\sigma)$ and $c(\tau)$ are the extremities of the segment shared by the two squares in the 2-ball $f_{7}$; and, thus, such a segment is not an edge in $\mathcal{E}_{S}$ by definition.

This shows that $\mathcal{F}_{S}, \mathcal{E}_{S}$ and $\mathcal{P}_{S}$ are families of 2-balls, 1-balls and 0-balls, respectively. To prove that $\mathcal{C}_{S}=\left(\mathcal{F}_{S}, \mathcal{E}_{S}, \mathcal{P}_{S}\right)$ is a cellular decomposition we start with Property (4). This property follows from the next technical result since a xel $\sigma \in \operatorname{cell}_{3}\left(R^{3}\right)$ belongs to $S$ if and only if $\sigma \in \operatorname{supp}(S)$.

Proposition 5. Let $O$ be a digital object in the device model $R^{3}$ and $\gamma \in R^{3} a$ cell in $\operatorname{supp}(O)$. Then, at least one of the vertices $\alpha \leq \gamma$ is maximal with respect to $O$.

By Property (4) it suffices to check that $\left|\mathcal{A}_{S}\right| \subseteq \cup\left\{f_{\alpha} ; f_{\alpha} \in \mathcal{F}_{S}\right\}$ to obtain Property (3); and this can be also straightforwardly proved from Proposition 5 For the proof of Property (1), let $f_{\alpha}, f_{\beta} \in \mathcal{F}_{S}$ be two distinct faces such that $f_{\alpha} \cap f_{\beta} \neq \emptyset$. By condition (4) in Definition 5 the traces $\operatorname{st}_{3}(\alpha ; S)$ and $\operatorname{st}_{3}(\beta ; S)$ share at most two xels of $S$; and, thus, Property (1) follows by the definition of the family of edges $\mathcal{E}_{S}$. And, if $e_{\alpha_{1} \beta_{1}}=f_{\alpha_{1}} \cap f_{\beta_{1}}$ and $e_{\alpha_{2} \beta_{2}}=f_{\alpha_{2}} \cap f_{\beta_{2}}$ are two distinct edges, the set $\left\{f_{\alpha_{1}}, f_{\beta_{1}}, f_{\alpha_{2}}, f_{\beta_{2}}\right\}$ contains at least three different elements; and, then, condition (3) in Definition 5 yields Property (2).

Finally, from conditions (4) and (5) in Definition 5 is not difficult to show that the cellular decomposition $\mathcal{C}_{S}$ satisfy also Properties (5) and (6), and thus $\left|\mathcal{A}_{S}\right|$ is a combinatorial manifold.

\section{References}

1. R. Ayala, E. Domínguez, A.R. Francés, A. Quintero. Digital Lighting Functions. Lecture Notes in Computer Science. 1347 (1997) 139-150.

2. R. Ayala, E. Domínguez, A.R. Francés, A. Quintero. Weak Lighting Functions and Strong 26-surfaces. To appear in Theoretical Computer Science.

3. R. Ayala, E. Domíguez, A. R. Francés and A. Quintero. A Digital Index Theorem. Int. J. Patter Recog. Art. Intell. 15(7) (2001) 1-22.

4. G. Bertrand, R. Malgouyres. Topological Properties of Discrete Surfaces. Lect. Notes in Comp. Sciences. 1176 (1996) 325-336.

5. M. Couprie, G. Bertrand. Simplicity Surfaces: a new definition of surfaces in $\mathbb{Z}^{3}$. SPIE Vision Geometry V. 3454 (1998) 40-51. 
6. R. Malgouyres, G. Bertrand. Complete Local Characterization of Strong 26Surfaces: Continuous Analog for Strong 26-Surfaces. Int. J. Pattern Recog. Art. Intell. 13(4) (1999) 465-484.

7. T.Y. Kong, A.W. Roscoe. Continuous Analogs of Axiomatized Digital Surfaces. Comput. Vision Graph. Image Process. 29 (1985) 60-86.

8. T.Y. Kong, A. Rosenfeld. Digital Topology: Introduction and Survey. Comput. Vision Graph. Image Process. 48 (1989) 357-393.

9. D.G. Morgenthaler, A. Rosenfeld. Surfaces in three-dimensional Digital Images. Inform. Control. 51 (1981) 227-247.

10. C.P. Rourke, and B.J. Sanderson. Introduction to Piecewise-Linear Topology. Ergebnisse der Math. 69, Springer 1972. 